\title{
293 Thyroglossal cysts and sinuses
}

A Result from developmental abnormalities of the descent of the thyroid from the foramen caecum.

B Usually present as a midline neck swelling in a child or young adult.

C The majority are above the level of the hyoid.

D An infected cyst should be incised and drained.

E In Sistrunk's operation, the entire hyoid bone is removed.

\section{Branchial sinuses and fistulae}

A The majority are present at birth.

B Internal opening in the supratonsillar fossa implies origin from the cervical sinus of His.

C A tract passing between internal and external carotid arteries suggests origin from the first branchial cleft.

D A collaural fistula has two openings.

E First line treatment consists of injection of sclerosants.

\section{Branchial cysts}

A Most are associated with internal openings.

B Histology supports the theory of origin from squamous cell rests within lymph nodes.

C Peak age of onset is 20-30 years.

D Aspiration should never be performed because of the risk of implanting cholesterol crystals in the neck.

E Hodgkin's disease is part of the differential diagnosis. 